\title{
Endoscopic full-thickness resection (eFTR) of colorectal lesions: results from the Dutch colorectal eFTR registry
}

\author{
Authors \\ Liselotte W. Zwager ${ }^{1,}{ }^{,}$, Barbara A. J. Bastiaansen ${ }^{1,}{ }^{*}$, Maxime E. S. Bronzwaer ${ }^{1}$, Bas W. van der Spek ${ }^{2}$, G. Dimitri N. \\ Heine $^{2}$, Krijn J. C. Haasnoot ${ }^{3}$, Hedwig van der Sluis ${ }^{4}$, Lars E. Perk ${ }^{5}$, Jurjen J. Boonstra ${ }^{6}$, Svend T. Rietdijk ${ }^{7}$, Hugo J. \\ Wolters $^{8}$, Bas L. A. M. Weusten ${ }^{9}$, Lennard P. L. Gilissen ${ }^{10}$, W. Rogier ten Hove ${ }^{11}$, Wouter B. Nagengast ${ }^{12}$, Frank C. \\ Bekkering ${ }^{13}$, M. P. Schwartz ${ }^{14}$, Jochim S. Terhaar sive Droste ${ }^{15}$, Marije S. Vlug ${ }^{16}$, Martin H. M. G. Houben ${ }^{17}$, Francisco J. \\ Rando Munoz ${ }^{18}$, Tom C. J. Seerden ${ }^{19}$, Hanneke Beaumont ${ }^{20}$, Rogier de Ridder ${ }^{21}$, Evelien Dekker ${ }^{1}$, Paul Fockens ${ }^{1}$, on \\ behalf of the Dutch eFTR Group
}

Institutions

1 Department of Gastroenterology and Hepatology, Amsterdam University Medical Centers, location AMC, Amsterdam Gastroenterology \& Metabolism, University of Amsterdam, Amsterdam, The Netherlands

2 Department of Gastroenterology and Hepatology, Noordwest Hospital Group, Alkmaar, The Netherlands

3 Department of Gastroenterology and Hepatology, University Medical Center Utrecht, Utrecht, The Netherlands

4 Department of Gastroenterology and Hepatology, Isala Clinics, Zwolle, The Netherlands

5 Department of Gastroenterology and Hepatology, Haaglanden Medical Center, The Hague, The Netherlands

6 Department of Gastroenterology and Hepatology, Leiden University Medical Center, Leiden, The Netherlands

7 Department of Gastroenterology and Hepatology, Onze Lieve Vrouwe Gasthuis, Amsterdam, The Netherlands

8 Department of Gastroenterology and Hepatology, Martini Hospital, Groningen, The Netherlands

9 Department of Gastroenterology and Hepatology, St. Antonius Hospital, Nieuwegein, The Netherlands

10 Department of Gastroenterology and Hepatology, Catharina Hospital, Eindhoven, The Netherlands

11 Department of Gastroenterology and Hepatology, Alrijne Medical Group, Leiden, The Netherlands

12 Department of Gastroenterology and Hepatology, University Medical Center Groningen, Groningen, The Netherlands

13 Department of Gastroenterology and Hepatology, IJsselland Hospital, Capelle aan den IJssel, The Netherlands

14 Department of Gastroenterology and Hepatology, Meander Medical Center, Amersfoort, The Netherlands

15 Department of Gastroenterology and Hepatology, Jeroen Bosch Hospital, Den Bosch, The Netherlands
16 Department of Gastroenterology and Hepatology, Dijklander Hospital, Hoorn, The Netherlands

17 Department of Gastroenterology and Hepatology, Haga Teaching Hospital, The Hague, The Netherlands

18 Department of Gastroenterology and Hepatology, Nij Smellinghe Hospital, Drachten, The Netherlands

19 Department of Gastroenterology and Hepatology, Amphia Hospital, Breda, The Netherlands

20 Department of Gastroenterology and Hepatology, Amsterdam University Medical Center, location VU, Amsterdam, The Netherlands

21 Department of Gastroenterology and Hepatology, Maastricht University Medical Center, Maastricht, The Netherlands

submitted 3.2.2020

accepted after revision 22.4.2020

published online 3.6 .2020

Bibliography

Endoscopy 2020; 52: 1014-1023

DOI 10.1055/a-1176-1107

ISSN 0013-726X

(c) 2020. Thieme. All rights reserved.

Georg Thieme Verlag KG, Rüdigerstraße 14,

70469 Stuttgart, Germany

Corresponding author

Barbara Bastiaansen, MD, Department of Gastroenterology and Hepatology, Amsterdam University Medical Centers, location AMC, Meibergdreef 9, 1105 AZ Amsterdam, The Netherlands

b.a.bastiaansen@amsterdamumc.nl

$\circledast$ Supplementary material

Online content viewable at:

https://doi.org/10.1055/a-1176-1107

\footnotetext{
* Contributed equally to this article
} 
Scan this QR-Code for the author commentary.

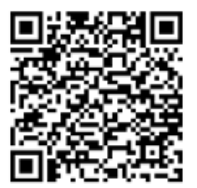

\section{ABSTRACT}

Background Endoscopic full-thickness resection (eFTR) is a minimally invasive resection technique that allows definite diagnosis and treatment for complex colorectal lesions $\leq 30 \mathrm{~mm}$ unsuitable for conventional endoscopic resection. This study reports clinical outcomes from the Dutch colorectal eFTR registry.

Methods Consecutive patients undergoing eFTR in 20 hospitals were prospectively included. The primary outcome was technical success, defined as macroscopic complete en bloc resection. Secondary outcomes were: clinical success, defined as tumor-free resection margins ( $R 0$ resection); full-thickness resection rate; and adverse events.
Results Between July 2015 and October 2018, 367 procedures were included. Indications were difficult polyps (nonlifting sign and/or difficult location; $n=133$ ), primary resection of suspected T1 colorectal cancer (CRC; $n=71$ ), reresection after incomplete resection of T1 CRC $(n=150)$, and subepithelial tumors $(n=13)$. Technical success was achieved in 308 procedures (83.9\%). In 21 procedures (5.7\%), eFTR was not performed because the lesion could not be reached or retracted into the cap. In the remaining 346 procedures, R0 resection was achieved in 285 (82.4\%) and full-thickness resection in 288 (83.2\%). The median diameter of resected specimens was $23 \mathrm{~mm}$. Overall adverse event rate was $9.3 \%(n=34 / 367)$ : 10 patients $(2.7 \%)$ required emergency surgery for five delayed and two immediate perforations and three cases of appendicitis.

Conclusion eFTR is an effective and relatively safe en bloc resection technique for complex colorectal lesions with the potential to avoid surgery. Further studies assessing the role of eFTR in early CRC treatment with long-term outcomes are needed.

\section{Introduction}

Endoscopic full-thickness resection (eFTR) is an emerging innovative endoscopic resection technique for complex colorectal lesions. With the advantage of enabling a transmural resection, eFTR offers an alternative to radical surgery in lesions considered incurable with current resection techniques such as endoscopic mucosal resection (EMR) or endoscopic submucosal dissection (ESD).

In clinical practice, the main indications for eFTR are nonlifting lesions with severe submucosal fibrosis, lesions that involve difficult anatomical locations such as the appendiceal orifice or diverticula, and subepithelial tumors. Furthermore, eFTR is gaining interest as a valid diagnostic and therapeutic treatment option for $\mathrm{T} 1$ colorectal cancer (CRC), as it can provide high quality pathological specimens and exact histological risk assessment [1].

Several studies have reported encouraging results on the short-term safety and efficacy of eFTR for numerous indications [1-12]. However, firm conclusions on clinical results will require analysis of large prospective series of patients in everyday clinical practice.

Since the introduction of the full-thickness resection device (FTRD; Ovesco Endoscopy AG, Tübingen, Germany) in the Netherlands in 2015, we started a nationwide prospective registry of all consecutive eFTR procedures to monitor patient outcomes and further increase knowledge on clinical applicability and safety. In this prospective observational multicenter study, we aimed to evaluate the technical and clinical success, and safety of eFTR for colorectal lesions in current clinical practice.

\section{Methods}

\section{Study design}

In this study, all registered procedures between July 2015 and October 2018 were analyzed. Results were prospectively recorded in 20 Dutch hospitals (5 academic and 15 non-academic). Procedures were performed by eFTR-certified gastroenterologists. For eFTR certification, experienced gastroenterologists attended a 1-day training course on eFTR, with thorough theoretical background and hands-on training in ex vivo porcine models.

Because the data were collected as part of standard medical care, the Institutional Review Board of the Amsterdam University Medical Centers decided that the study fell outside of the legislation regarding Medical Research Involving Human Subjects Act and therefore formal ethical approval was not deemed necessary. The study was registered in the Dutch Trial Register: NTR6292 (http://www.trialregister.nl/).

All patients were informed about the eFTR procedure and the peri-procedural risks by their treating physician and informed consent for the procedure was obtained. The results of 58 eFTR procedures were published previously $[13,14]$. Coded data were collected into a secure online structured database provided by Castor EDC, Amsterdam, the Netherlands [15].

\section{Outcome}

The primary outcome was the technical success of all initiated procedures, defined as the proportion of macroscopic complete en bloc resections (judged by the endoscopist).

Secondary outcome measures included: clinical success, defined as the rate of histologically confirmed radical (RO) resections (tumor-free lateral and deep resection margins); rate of 

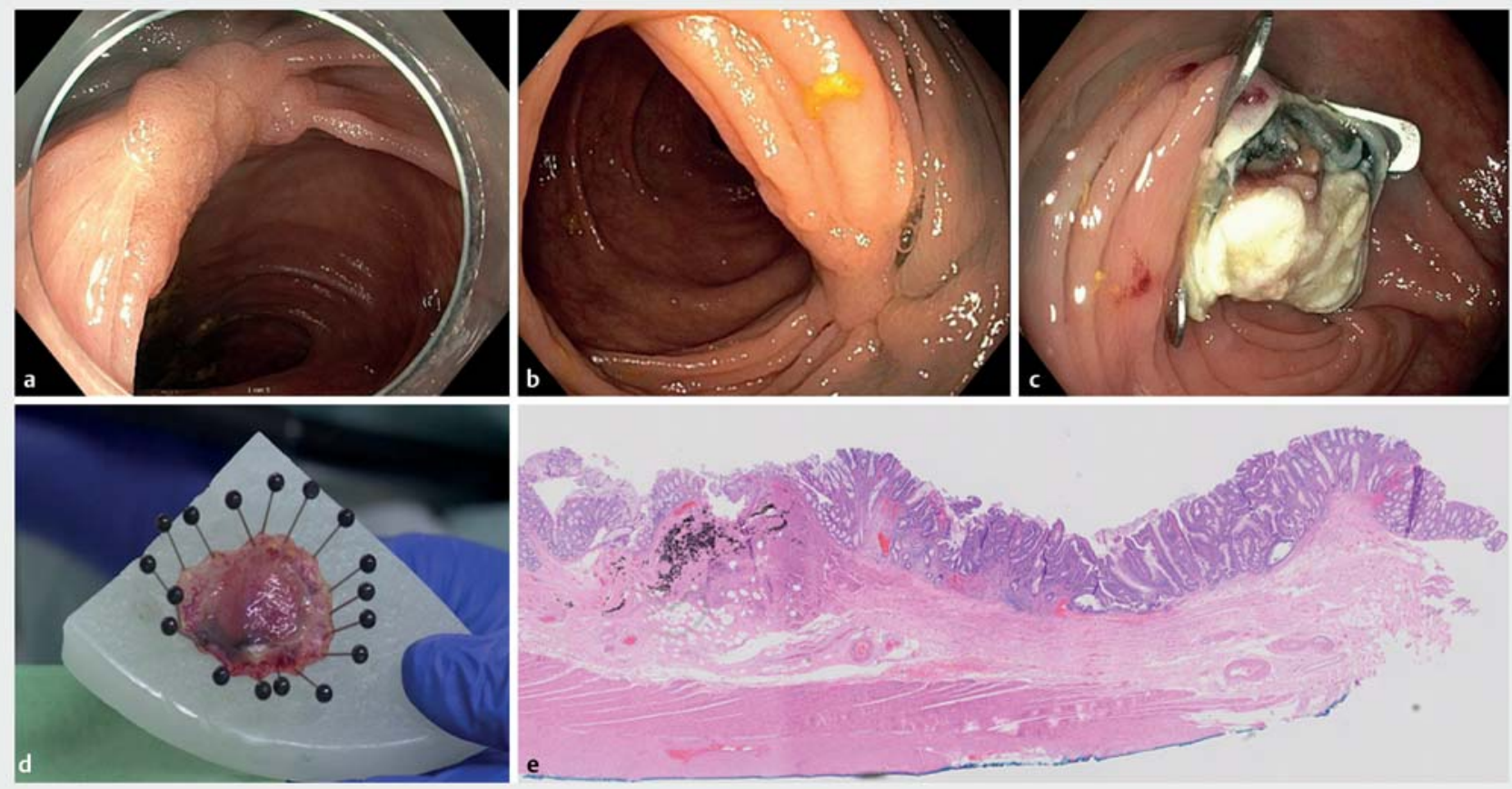

- Fig. 1 Endoscopic full-thickness resection of a recurrent non-lifting adenoma (third recurrence). a-c Endoscopic images showing: a,b a recurrent adenoma in the transverse colon with clear fibrosis and fold conversion; $\mathbf{c}$ the resection site with the over-the-scope clip in place and exposure of the submucosal tattoo. $\mathbf{d}$ Macroscopic appearance of the resected specimen pinned down with the serosal side up (plus tattoo) on paraffin. e Histopathological appearance showing a full-thickness resection of a tubulovillous adenoma with low grade dysplasia, with clear submucosal fibrosis and ink. Lateral and deep resection margins were clear. Source for Fig. 1e: Lianne Koens.

histologically confirmed full-thickness resection (presence of the muscularis propria in the resection specimen); procedurerelated adverse events; and recurrence at first follow-up colonoscopy.

\section{Study subjects}

Eligibility for eFTR was judged by the treating physician after a complete colonoscopy had been performed. Indications for eFTR were "difficult polyps," including non-lifting polyps (treatment naïve, recurrent, or incompletely resected polyps) and polyps involving the appendiceal orifice or a diverticulum; primary resection for suspected T1 CRCs; and secondary completion treatment after previous incomplete endoscopic resection with a positive $(\leq 1 \mathrm{~mm})$ or non-assessable resection margin (R1/Rx resection). Other indications for eFTR were subepithelial tumours. Because of the limited size of the FTRD cap, lesions with an estimated diameter of $\geq 30 \mathrm{~mm}$ were considered unsuitable for eFTR [16]. As this study used a prospective registry of current clinical practice, no explicit exclusion criteria were formulated.

\section{eFTR procedure and management}

All patients received standard split-dose polyethylene glycol (PEG) bowel preparation. Procedures were performed with the patient under sedation with propofol or midazolam and/or fentanyl, according to local practice. A single dose of prophylactic intravenous antibiotic therapy was advised for all eFTR proce- dures in the early study period, but since 2017 antibiotic prophylaxis was deemed unnecessary and was no longer advised, except for appendiceal lesions without previous appendectomy. Patients on anticoagulation therapy were advised to use their medication according to the Dutch guideline for antithrombotic therapy in endoscopy: coumarins and direct-acting oral anticoagulants (DOACs) were discontinued, single-agent antiplatelet agents could be continued [17]. The FTRD was used in all procedures, and these were performed using $\mathrm{CO}_{2}$ insufflation.

First, the target lesion was identified during a diagnostic endoscopy and marked by creating coagulation marks with the dedicated probe or by other means, and the lesion size was estimated at the discretion of the endoscopist. Thereafter, the colonoscope was withdrawn, the FTRD was mounted and the colonoscope was advanced again to the target area. A specialized grasping forceps was advanced through the working channel to grasp and slowly pull the lesion into the cap. Once the lateral margins of the lesion were pulled into the cap, the clip was deployed. Immediately thereafter, the tissue was resected with the pre-loaded snare, while the clip secured the integrity of the bowel wall. The endoscope with the resection specimen entrapped in the cap was then withdrawn. After the specimen had been secured and the device demounted, the endoscope was introduced once again to inspect the position of the clip and the resection site. The specimen was stretched and pinned onto cork or paraffin before immersion into formalin for histological analysis ( $\mathbf{F i g . 1}$ and $>$ Fig. 2) [13]. To obtain the most 

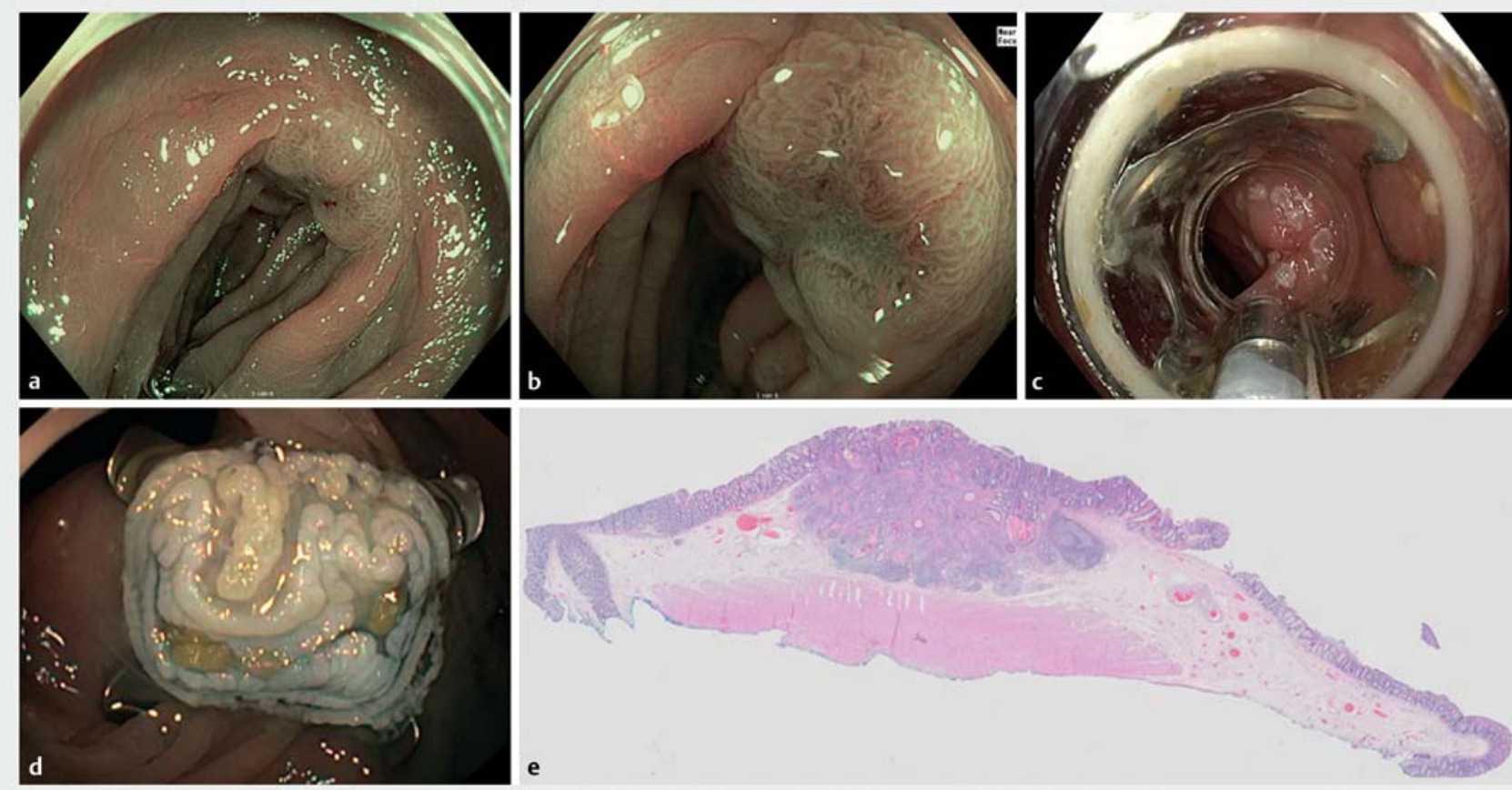

- Fig. 2 Completion endoscopic full-thickness resection after a previous incomplete resection of a low risk T1 colorectal cancer. a - $\mathbf{d}$ Endoscopic images showing: a,b narrow-band imaging of the target lesion; $\mathbf{c}$ the mounted full-thickness resection device on the marked lesion; $\mathbf{d}$ the full-thickness resection site with the over-the-scope clip. e Histopathological appearance showing a R0 resection of a moderately differentiated adenocarcinoma with deep submucosal invasion (SM3), without lymphovascular invasion or tumor budding. Source for Fig. 2e: Lianne Koens.

accurate measurement of lesion and specimen size after eFTR, we used the size measured at histology.

Clinical admission for 24 hours to monitor signs of discomfort, bleeding, or perforation was advised in the early study period, but from 2017 the advice was withdrawn and this was left to the discretion of treating physician. The recommended dietary regimen was a clear fluid diet for 24 hours, followed by a normal diet.

\section{Adverse events and follow-up}

All immediate procedure-related adverse events that resulted in prolonged admission and/or an intervention (i.e. blood transfusion, re-colonoscopy, or surgery) were recorded at the time of colonoscopy. Delayed procedure-related adverse events requiring readmission or intervention (i. e. blood transfusion, colonoscopy, or surgery) were recorded approximately 14 days after eFTR, when patients were contacted to discuss the histopathological results. The severity of adverse events was graded according to the American Society for Gastrointestinal Endoscopy [18].

Patients were scheduled for follow-up colonoscopy after 36 months. The eFTR scar was inspected with high definition white-light endoscopy and (digital) chromoendoscopy for macroscopic recurrent or residual tissue and the presence of the clip. Endoscopic and/or histological findings compatible with granulation tissue or reactive changes were left untreated. Residual polyp tissue was treated with conventional treatment strategies if possible. When endoscopic resection of the resi- dual polyp was considered impossible or submucosal invasive cancer was suspected, a tattoo (SPOT) was placed followed by case discussion in a colorectal multidisciplinary meeting. Follow-up colonoscopies were scheduled depending on histological and endoscopic findings. Patients referred for additional surgery after eFTR were excluded from scar surveillance.

\section{Statistical analysis}

Standard descriptive statistics were used. Variables are reported as mean with standard deviation (SD) for continuous and normally distributed variables, as median and interquartile range (IQR) for non-normally distributed continuous variables, and as percentages for counts or categorical variables. Categorical variables were analyzed using chi-squared or two-sided Fisher's exact tests. A two-sided $P$ value $<0.05$ was considered statistically significant. Statistical analysis was performed using SPSS 24 (SPSS, Chicago, Illinois, USA).

\section{Results}

\section{Patients characteristics, procedural data, and technical success}

A total of 367 eFTR procedures were performed in 362 patients between July 2015 and October 2018 and were included for analysis (63\% men; mean age 69 years). The indications were: difficult polyps $(n=133)$, primary resection of suspected T1 CRC $(n=71)$, re-resection after previous incomplete resection of T1 CRC $(n=150)$, and subepithelial tumors $(n=13)$. Proce- 
- Table 1 Characteristics of the 362 patients who underwent endoscopic full-thickness resection (eFTR) and the 367 procedures performed.

\begin{tabular}{|c|c|}
\hline sex, male, n (\%) & $227(62.7)$ \\
\hline mean age $(S D)$, years & $69(8.2)$ \\
\hline \multicolumn{2}{|l|}{ Indication, $\mathrm{n}$ (\% of eFTR procedures) } \\
\hline - T1 colorectal cancer & $221(60.2)$ \\
\hline - Primary treatment & $71(19.3)$ \\
\hline - Secondary treatment & $150(40.9)$ \\
\hline - Difficult polyp & $133(36.2)$ \\
\hline - Recurrence or incomplete resection & $85(23.2)$ \\
\hline - Primary treatment for polyp with non-lifting sign & $28(7.6)$ \\
\hline - Polyps involving a diverticulum & $5(1.4)$ \\
\hline - Polyps involving the appendix & $15(4.1)$ \\
\hline - Preceding appendectomy & $3(20.0)$ \\
\hline - Subepithelial tumor & $13(3.5)$ \\
\hline Estimated median diameter of lesion (IQR), mm* & $10(8-15)$ \\
\hline \multicolumn{2}{|l|}{ Lesion location, $\mathrm{n}$ (\% of eFTR procedures) } \\
\hline - Proximal (cecum to splenic flexure) & $143(39.0)$ \\
\hline - Cecum & $27(7.4)$ \\
\hline - Appendix & $17(4.6)$ \\
\hline - Ascending colon & $51(13.9)$ \\
\hline - Hepatic flexure & $14(3.8)$ \\
\hline - Transverse colon & $27(7.4)$ \\
\hline - Splenic flexure & $7(1.9)$ \\
\hline - Distal (descending colon to rectum) & $224(61.0)$ \\
\hline - Descending colon & $22(6.0)$ \\
\hline - Sigmoid & $123(33.5)$ \\
\hline - Rectum & 79 (21.5) \\
\hline $\begin{array}{l}\text { SD, standard deviation; IQR, interquartile range. } \\
\text { * Size estimated by endoscopist. }\end{array}$ & \\
\hline
\end{tabular}

dures were performed by 37 certified endoscopists divided over 20 Dutch hospitals. Patient characteristics are shown in - Table 1.

Technical success was achieved in $83.9 \%$ of all procedures $(n=308 / 367)$ ( $\triangleright$ Table 2$)$. In $1.9 \%$ of procedures $(7 / 367)$, the target lesion could not be reached successfully ( $>$ Fig. 3 ). The main reason for not reaching the lesion was failure to pass the sigmoid colon with the FTRD in three procedures (42.9\%); for the other four procedures, no reason was described. In 14 procedures (3.9\%), the eFTR was not performed because the lesion could not be retracted into the cap owing to lack of mobility of the tissue. Of these 14 lesions, 12 were requiring re-resection after previous EMR.

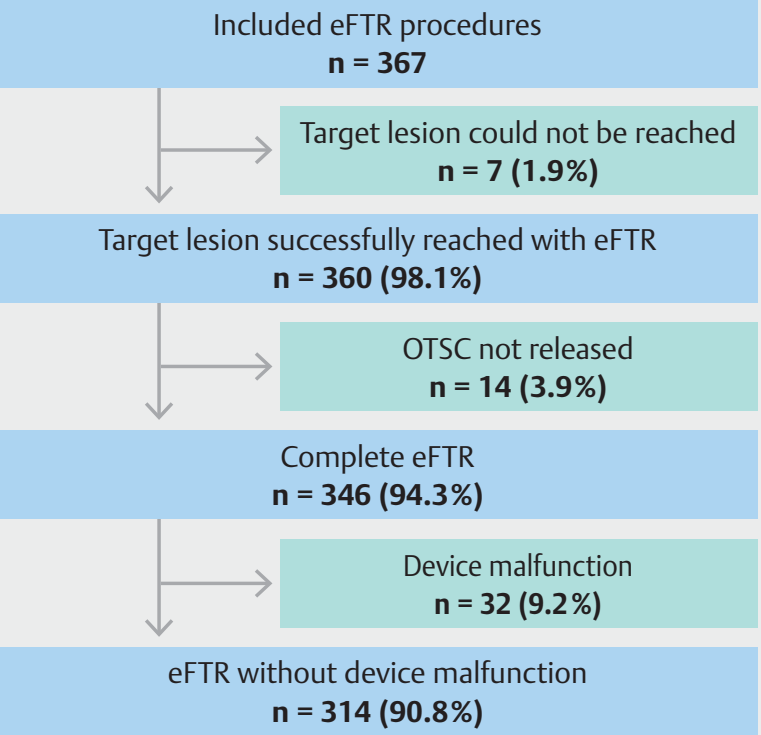

- Fig. 3 Flowchart of the endoscopic full-thickness resection (eFTR) procedures included in the study. OTSC, over-the-scope clip.

In 346 procedures ( $94.3 \%$ ), it was possible to perform eFTR (lesion reached and clip deployed) and histology was obtained. Device malfunctions were reported in 32 of these 346 procedures $(9.2 \%)$. These were caused by snare malfunctions $(n=$ $23 ; 6.6 \%)$, grasper dysfunction ( $n=6 ; 1.7 \%)$, and inability to release the clip ( $n=3 ; 0.9 \%)$. However, it was possible to complete the resection in 22 of the snare malfunctions and five of the grasper dysfunctions.

\section{Clinical success}

In the total cohort of procedures amenable to eFTR $(n=346)$, histological R0 resection was achieved in 285 procedures (82.4\%) and full-thickness resection in 288 (83.2\%) (> Table 2 ). There were 14 cases that were judged during the procedure to be technically unsuccessful owing to macroscopic incomplete resection but were found to have tumor-free resection margins (R0) at histology (4.0\%). The median diameters of the resected lesions and the resected specimens at histology were $12 \mathrm{~mm}$ (IQR 8-17) and $23 \mathrm{~mm}$ (IQR 20-28), respectively. R0 resection for lesions $>20 \mathrm{~mm}$ was achieved in $72.7 \%$ vs. $78.3 \%$ for lesions $\leq 20 \mathrm{~mm}(P=0.71)$.

eFTR in the subgroup of T1 CRCs $(n=221)$ was technical successful in 191 procedures (86.4\%) and R0 resection was achieved in 186 procedures (88.2\%). For primary resections of T1 CRCs, the R0 resection rate was $77.9 \%(n=53)$ compared with $93.0 \%(n=133)$ for secondary treatment (Table 1s).

After resection of the scar for previously incompletely resected T1 CRCs (R1/Rx), only scar tissue was found at histology in 117 procedures (81.8\%). Residual adenocarcinoma was found in 11 procedures (7.7\%). Of these, five patients were referred for additional oncological surgery because of high risk features for lymph node metastasis (LNM) or an incomplete 
- Table 2 Technical success rates for all endoscopic full-thickness resection (eFTR) procedures initiated and clinical success rates in those amenable to EFTR.

\begin{tabular}{|c|c|c|c|c|}
\hline & Overall & T1 CRCs & Difficult polyps & $\begin{array}{l}\text { Subepithelial } \\
\text { tumors }\end{array}$ \\
\hline Initiated eFTR procedures, $\mathrm{n}$ & 367 & 221 & 133 & 13 \\
\hline Technical success, $\mathrm{n}(\%)$ & $308(83.9)$ & $191(86.4)$ & $105(78.9)$ & $12(92.3)$ \\
\hline Procedures amenable to eFTR, $\mathrm{n}^{1}$ & 346 & 211 & 122 & 13 \\
\hline \multicolumn{5}{|l|}{ Resection, n (\%) } \\
\hline - Ro & $285(82.4)$ & $186(88.2)$ & $86(70.5)$ & $13(100)$ \\
\hline - Full-thickness & $288(83.2)$ & $176(83.4)$ & $100(82.0)$ & $12(92.3)$ \\
\hline \multicolumn{5}{|l|}{ Lesion diameter, median (IQR), mm² } \\
\hline - Lesion & $12(8-17)$ & $13(9-18)$ & $12(8-15)$ & $9(5-15)$ \\
\hline - Resected specimen & $23(20-28)$ & $23(19-27)$ & $23(20-29)$ & $26(20-30)$ \\
\hline \multicolumn{5}{|l|}{ Location lesion, $n(\%)^{3}$} \\
\hline - Proximal & $131(37.9)$ & $55(26.1)$ & $76(62.3)$ & $0(0)$ \\
\hline - Distal & $215(62.1)$ & $156(73.9)$ & $46(37.7)$ & $13(100)$ \\
\hline \multicolumn{5}{|l|}{ Histology, n (\%) } \\
\hline - Normal colon tissue/scar & $145(41.9)$ & $120(56.9)$ & $16(13.1)$ & $8(61.5)$ \\
\hline - Adenoma with LGD & $75(21.7)$ & $13(6.2)$ & $63(51.6)$ & $0(0)$ \\
\hline - Adenoma with HGD & $15(4.3)$ & $5(2.4)$ & $10(8.2)$ & $0(0)$ \\
\hline - Serrated polyp & $17(4.9)$ & $3(1.4)$ & $14(11.5)$ & $0(0)$ \\
\hline - Adenocarcinoma & $84(24.3)$ & $67(31.8)$ & $17(13.9)$ & $0(0)$ \\
\hline - Neuroendocrine tumor & $5(1.4)$ & $0(0)$ & $0(0)$ & $5(38.5)$ \\
\hline - Other $^{4}$ & $3(0.9)$ & $3(1.4)$ & $0(0)$ & $0(0)$ \\
\hline - No pathology & $2(0.6)$ & $0(0)$ & $2(1.6)$ & $0(0)$ \\
\hline \multicolumn{5}{|c|}{$\begin{array}{l}\text { CRC, colorectal cancer; IQR, interquartile range; LGD, low grade dysplasia; HGD, high grade dysplasia. } \\
{ }^{1} \text { Ability to obtain histology (lesion was reached and over-the-scope clip deployed). } \\
{ }^{2} \text { Size measured by the pathologist at histopathology. } \\
3 \text { Proximal is defined as cecum to splenic flexure and distal as descending colon to rectum. }\end{array}$} \\
\hline
\end{tabular}

eFTR. In one of these five patients, surgery was not performed owing to significant comorbidities. Of the four operated patients, one surgical specimen showed residual T2 cancer without LNM and one a T2 cancer with a positive lymph node. In the two other patients, no residual cancer was found.

In the 68 patients with a primary resection for T1 CRC, 18 (26.5\%) were referred for secondary surgery because of high risk features and/or incomplete resection. Of all the surgical specimens, one case had residual cancer (T3N2) (Table 2s).

In the subgroup of difficult polyps $(n=133)$, the procedure was technically successful in 105 procedures $(78.9 \%)$ and an R0 resection was obtained in 86 procedures $(70.5 \%)$. In the subepithelial lesions $(n=13)$, eFTR was technically successful in 12 cases $(92.3 \%)$ and $R 0$ resection was achieved in all cases ( Table 2).

\section{Adverse events}

Overall, adverse events occurred in 34 procedures $(9.3 \%)(>\mathrm{Ta}$ ble 3$)$. Severe adverse events occurred in 10 procedures $(2.7 \%)$, including seven perforations (1.9\%). In two patients, the perforation was noticed immediately; in both, the clip had not been released appropriately before the lesion was resected. In five patients delayed perforations occurred on days $1-8$. In three of these, the lesion was located in the sigmoid and one of these patients was on immunosuppressive therapy for inflammatory bowel disease. The other two delayed perforations were located in the transverse colon and cecum. All seven patients needed surgical repair. In addition, three patients with an appendiceal lesion ( $n=3 / 15 ; 20.0 \%$ ) developed secondary appendicitis (all without previous appendectomy) and underwent an acute laparoscopic appendectomy $(n=2)$ or a laparoscopic ileocecal resection $(n=1)$. 
- Table 3 Safety of the 367 endoscopic full-thickness resection (eFTR) procedures.

\begin{tabular}{|c|c|}
\hline Adverse events, $\mathbf{n}(\%)$ & $34(9.3)$ \\
\hline Mild adverse events ${ }^{1}$ & $16(4.4)$ \\
\hline - Bleeding & $8(2.2)$ \\
\hline - Perforation ( 1 immediate / 1 delayed) & $2(0.5)$ \\
\hline - Post-polypectomy syndrome & $1(0.3)$ \\
\hline - Urinary retention & $1(0.3)$ \\
\hline - Abdominal pain & $2(0.5)$ \\
\hline - Periappendiceal abscess & $1(0.3)$ \\
\hline - Collapse & $1(0.3)$ \\
\hline Moderate adverse events ${ }^{2}$ & $8(2.2)$ \\
\hline - Bleeding & $7(1.9)$ \\
\hline - Abdominal pain & $1(0.3)$ \\
\hline Severe adverse events ${ }^{3}$ & $10(2.7)$ \\
\hline - Perforation ( 2 immediate / 5 delayed) & $7(1.9)$ \\
\hline - Appendicitis & $3(0.8)$ \\
\hline Indication for surgery, n (\%) & $65(17.7)$ \\
\hline - No eFTR performed & $13(3.5)$ \\
\hline - R1/Rx eFTR resection & $11(3.0)$ \\
\hline - One or more high risk CRC features & $18(4.9)$ \\
\hline - Adverse events & $10(2.7)$ \\
\hline - Perforation & $7(70.0)$ \\
\hline - Appendicitis & $3(30.0)$ \\
\hline - No surgery due to comorbidities & $7(1.9)$ \\
\hline - Other ${ }^{4}$ & $6(1.6)$ \\
\hline Median hospital stay (IQR), days & $1.0(1-1)$ \\
\hline \multicolumn{2}{|c|}{$\begin{array}{l}\text { CRC, colorectal cancer; IQR, interquartile range. } \\
1 \text { Mild: unplanned hospital admission or prolongation of hospital stay for } \leq 3 \\
\text { days or post-procedure medical consultation or procedure aborted (or not } \\
\text { started) because of an adverse event. }\end{array}$} \\
\hline \multicolumn{2}{|c|}{$\begin{array}{l}2 \text { Moderate: unplanned hospital admission or prolongation of hospital stay } \\
\text { for } 4-10 \text { days or intensive care admission for } 1 \text { night or radiological inter- } \\
\text { vention or transfusion or repeat endoscopy for an adverse event or un- } \\
\text { planned anesthesia/ventilation support, i. e. endotracheal intubation dur- } \\
\text { ing conscious sedation. }\end{array}$} \\
\hline \multicolumn{2}{|c|}{$\begin{array}{l}{ }^{3} \text { Severe: unplanned hospital admission or prolongation of hospital stay for }> \\
10 \text { days or intensive care admission for }>1 \text { night or surgery for an adverse } \\
\text { event or permanent disability [18]. }\end{array}$} \\
\hline \multicolumn{2}{|c|}{$\begin{array}{l}{ }^{4} \text { Surgical resection was due to a synchronous CRC }(n=2) \text {, another polyp that } \\
\text { could not be endoscopically resected }(n=1) \text {, preference for a surgical re- } \\
\text { section }(n=1) \text {, and the presence of mucin fields }(n=1) \text {. One patient parti- } \\
\text { cipated in a trial and also received chemotherapy. }\end{array}$} \\
\hline
\end{tabular}

Moderately severe adverse events were observed following eight procedures $(2.2 \%)$. In seven (1.9\%), post-procedural bleeding was observed that required re-admission or repeat endoscopy. In 16 (4.4\%), a mild adverse event was observed. In two cases $(0.5 \%)$, this was due to a perforation. The first perforation, which was seen immediately after the procedure, could be successfully clipped with an over-the-scope clip (OTSC). The second case, located in the sigmoid, occurred a few days after an uneventful eFTR and was treated conservatively with antibiotics and re-admission for 3 days. One patient with a history of appendectomy developed a peri-appendiceal abscess after eFTR for an appendiceal lesion and was treated conservatively.

\section{Surgery}

Overall, 65 patients (17.7\%) underwent surgery after the initial eFTR procedure ( $\triangleright$ Table 3 ). In 13 (3.5\%), surgery was required because eFTR could not be performed (lesion not reached or clip not deployed); in 10 (2.7\%), emergency surgery was performed because of an adverse event. Elective oncological surgery was performed in 29 patients (7.9\%): in 11 (3.0\%) after incomplete eFTR (R1/Rx), and in 18 (4.9\%) because of high risk features for LNM. Of these elective surgery cases, histology was available in 26 (89.7\%). Residual adenocarcinoma was found in three (11.5\%) (Table $2 \mathrm{~s}$ ).

\section{Follow-up}

Endoscopic follow-up data were available for 187 procedures (63.4\%) after eFTR and without additional surgery. Median time to follow-up was 4 months (IQR 3-6). In 31 procedures (16.6\%), the clip was still in situ at follow-up. In 100 procedures (33.9\%), endoscopic surveillance was still pending or not recorded. Furthermore, in the remaining eight cases (2.7\%), no followup was scheduled. Reasons to refrain from follow-up were comorbidities $(n=3)$, no indication for follow-up $(n=2)$, or unclear reasons $(n=3)$. Residual/recurrent lesions were seen in 12 patients (6.4\%). No adenocarcinomas were detected (Table 3s).

\section{Discussion}

In this study, we report the initial results of 367 eFTR procedures prospectively recorded in an eFTR registry in the Netherlands, representing the largest patient cohort published to date. While the interest and adoption of eFTR continues to grow amongst endoscopists, outcomes of large prospective series on its safety and efficacy are needed. In this multicenter collaboration, we aimed to investigate the clinical outcomes of eFTR for complex colorectal lesions.

In our study, the technical success rate for eFTR was $83.9 \%$ and $\mathrm{R} 0$ resection was achieved in $82.4 \%$ of the procedures. The results of our study are comparable with the results of the previous prospective study by Schmidt and colleagues, and to several other retrospective series [1-12]. In a meta-analysis comparing ESD and EMR for colorectal polyps, the R0 resection rate was $80.3 \%$ for ESD and $42.3 \%$ for EMR [19]. Therefore, R0 resection rates of eFTR are comparable with ESD and more favorable than EMR [20-25]. However, it is important to consider that this cohort mainly includes complex colorectal lesions, considered unsuitable for conventional endoscopic en bloc resection techniques. En bloc excision is always preferred as it provides a higher quality pathological specimen and lower recurrence rates. This advantage should however be balanced against a higher perforation risk [24]. eFTR can tackle the chal- 
lenge posed by submucosal fibrosis, which makes safe en bloc EMR or ESD difficult.

Although the learning curve for eFTR is acknowledged to be shorter than for ESD, eFTR remains a challenging procedure with several limitations [3]. At present, a consensus on appropriate patient selection for eFTR is lacking. The main limitation for colorectal eFTR is the limited lesion size that can be treated. The FTRD system accommodates lesions with a maximum diameter of $30 \mathrm{~mm}$ [16]. In contrast to Schmidt and colleagues, we did not find a significant difference in the $\mathrm{R} 0$ resection rate between lesions $>20 \mathrm{~mm}(72.7 \%)$ vs. lesions $\leq 20 \mathrm{~mm}$ (78.3\%; $P$ $=0.71)[3,11]$. However, our subgroup of lesions larger $>20$ $\mathrm{mm}$ is relatively small and might have biased our results. Considering these results together with an average size of $23 \mathrm{~mm}$ for the resected specimen, we recommend lesions with a maximum estimated diameter of $20 \mathrm{~mm}$ appropriate for eFTR.

Further technical limitations of the device are the impaired visibility and tip flexibility due to the long cap. Using a "dummy" cap (prOVE cap; Ovesco Endoscopy AG) when it is suspected that introduction will be difficult or there is doubt about wall mobility could help to optimize patient selection. In our study, we report a relatively high rate of device malfunction (9.2\%). However, in the majority of cases (84\%), the procedure could be completed with clinical success. For this matter, we believe this rate of device malfunction is acceptable.

The most frequent indication for eFTR in our cohort was T1 CRC ( $n=221 ; 60.2 \%)$ [3]. Although literature is scarce, our R0 resection rate for $\mathrm{T} 1 \mathrm{CRC}$ of $88.2 \%$ compares favorably with previous studies [1,3].

In the group of patients with primary eFTR for T1 CRC, the R0 resection rate was $77.9 \%$ in our study. The retrospective study of Kuellmer and colleagues reported a lower $\mathrm{R} 0$ resection rate of $60.9 \%$ [1]. This difference in $\mathrm{R} 0$ resection rate could be explained by differences in the average size of the resected lesion, $20 \mathrm{~mm}$ in the German study vs. $14 \mathrm{~mm}$ in ours. Besides, in contrast to the prospective inclusion of lesions suspicious for T1 CRC in our study, Kuellmer and colleagues included mainly nonlifting lesions that were initially classified as benign but at histopathology were diagnosed as T1 CRC, suggesting the inclusion of more complex lesions [1].

We believe eFTR, having several benefits compared with ESD, can be considered a valid diagnostic and potentially therapeutic primary treatment option for T1 CRC. Besides the advantage of demanding less procedural skill and time than ESD, obtaining a transmural resection with eFTR optimizes histological assessment. Furthermore, eFTR enables safe and radical endoscopic resection of deep submucosal invasive cancer. Recent studies have reported a very low risk of LNM $(1 \%-2 \%)$ in T1 CRC, with deep submucosal invasion as the only risk factor for LNM [26, 27]. These findings underscore the need for clinical trials to assess the role of eFTR in T1 CRC treatment.

Resection of scars after previously incomplete $T 1$ CRC resection accounted for a large subgroup in our registry. Positive or indeterminate resection margins $(R 1 / R x)$ are associated with residual cancer $[28,29]$. Therefore, current guidelines advise oncological surgery after R1 / Rx resection, even in the absence of histological risk factors for LNM $[17,30]$. eFTR allows trans- mural resection of the scar with an $\mathrm{R} 0$ resection rate of $93.0 \%$, which is comparable with the $87.5 \%$ rate in Kuellmer's study [1]. Despite the fact only scar tissue and no residual cancer was found in $81.8 \%$ at histopathology, we believe eFTR can confirm local radicality by enabling a transmural scar excision or serve as a potentially curative completion treatment where there is residual cancer. However, currently the ability to histologically confirm complete scar excision is lacking, and therefore in clinical practice one is relying on the macroscopic completeness of scar resection. However, long-term outcomes are lacking and further data are therefore warranted.

The second most prevalent indication in our cohort was "difficult polyps," consisting of non-lifting polyps or polyps that were involving difficult locations. Overall, the R0 resection rate for this subgroup was $70.5 \%$, which was lower compared with the previously reported $\mathrm{R} 0$ rate of $77.7 \%$ in the study by Schmidt et al. [3]. However, we included mainly recurrent lesions after previously incomplete resections. As mentioned, submucosal scarring can limit adequate tissue mobilization into the cap $[6,11]$. From our experience, mobilization of recurrent lesions after previous EMR can be challenging. In 14 patients (3.9\%), eFTR could not be performed because the lesion could not be mobilized into the cap. Of these, 12 were scarred lesions previously treated with EMR. In certain cases, a dummy cap may help to improve patient selection.

Adverse events occurred in $9.3 \%$, with $2.7 \%$ being classified as severe. This severe adverse event rate requiring emergency surgery is comparable with previous studies (Schmidt et al., $2.2 \%$; Kuellmer et al., 3.8\%) [1,3]. Nevertheless, future efforts will hopefully further decrease complication rates. The most feared complication is a delayed perforation, which occurred in $1.6 \%$ of procedures and predominantly in the left-sided colon. Owing to partial wall excision, a relative stenosis can occur at the level of the clip. As fecal content is more solid in the distal colon, high pressures at the level of the clip could contribute to tissue disintegration or rupture. None of the three patients with delayed sigmoid perforation in our registry received post-procedural stool softeners. Although debatable, we believe prescribing post-procedural laxatives might reduce the delayed perforation risk in left-sided lesions.

Another cause of severe adverse event was appendicitis, occurring in $20.0 \%$ of procedures for appendiceal lesions without previous appendectomy. Closure of the remaining appendix by the clip carries a risk of acute appendicitis, most likely from retained mucus [13]. Schmidt and colleagues reported a lower secondary appendicitis rate of $8.8 \%$ [3]. There is no clear explanation for this discrepancy, although both are based on small subgroups. Larger cohorts with adequate follow-up are required to elucidate the position of eFTR in appendiceal lesions. In the meantime, patients should be well informed about the risk of secondary appendicitis.

Our study has several limitations that should be addressed. First, because this study is based on registry data, we cannot exclude potential selection bias and must rely on accurate data recording in all 20 participating centers. Second, follow-up data were not complete because not all surveillance colonoscopies had been performed or recorded yet. However, this registry 
started in a strong and transparent collaboration of 20 academic and non-academic centers and represents a good overview of eFTR for colorectal lesions in current clinical practice.

In conclusion, eFTR is an exciting innovative resection technique that is clinically feasible and safe for complex colorectal lesions $(\leq 20 \mathrm{~mm})$, with the potential to obviate the need for surgical resection. Further efficacy studies on eFTR as a primary and secondary treatment option for T1 CRC are needed, focusing on both the short- and long-term oncological results.

\section{Acknowledgments}

We thank all participating endoscopists of the eFTR working group for their efforts and cooperation and we want to thank dr. Lianne Koens, pathologist at Amsterdam UMC for providing the histology images.

A single unrestricted grant from Ovesco Endoscopy AG was received for database costs. Ovesco Endoscopy AG was not involved in the design, collection, analysis, or interpretation of our data. No other funding was received. No endoscopic equipment was donated by the manufacturer.

\section{Competing interests}

P. Fockens receives personal fees from Cook, Ethicon, and Olympus, and research support from Boston Scientific outside the submitted work. E. Dekker has endoscopic equipment on loan from FujiFilm, and has received a research grant from Fujifilm, consultancy fees from Fujifilm, Olympus, Tillotts, GI Supply, and CPP-FAP, and speaker's fees from Olympus, Roche, and GI Supply. B. Bastiaansen has received speaker's fees from Olympus, Tillotts Pharma AG, and Ovesco Endoscopy AG. The remaining authors declare that they have no conflict of interest.

Clinical trial

Trial Registration: Dutch Trial Register | Registration number (trial ID): NTR6292 | Type of study: Prospective study

\section{References}

[1] Kuellmer A, Mueller ], Caca K et al. Endoscopic full-thickness resection for early colorectal cancer. Gastrointest Endosc 2019; 89: 1180-1189 e1181

[2] Schmidt A, Bauerfeind P, Gubler C et al. Endoscopic full-thickness resection in the colorectum with a novel over-the-scope device: first experience. Endoscopy 2015; 47: 719-725

[3] Schmidt A, Beyna T, Schumacher B et al. Colonoscopic full-thickness resection using an over-the-scope device: a prospective multicentre study in various indications. Gut 2018; 67: 1280-1289

[4] Andrisani G, Pizzicannella M, Martino M et al. Endoscopic full-thickness resection of superficial colorectal neoplasms using a new overthe-scope clip system: A single-centre study. Dig Liver Dis 2017; 49: $1009-1013$

[5] Aepli P, Criblez D, Baumeler S et al. Endoscopic full thickness resection (EFTR) of colorectal neoplasms with the Full Thickness Resection Device (FTRD): Clinical experience from two tertiary referral centers in Switzerland. United European Gastroenterol J 2018; 6: 463-470
[6] Andrisani G, Soriani P, Manno M et al. Colo-rectal endoscopic fullthickness resection (EFTR) with the over-the-scope device (FTRD ${ }^{8}$ ): A multicenter Italian experience. Dig Liver Dis 2019; 51: 375-381

[7] Valli PV, Mertens J, Bauerfeind P. Safe and successful resection of difficult GI lesions using a novel single-step full-thickness resection device (FTRD $\circledast)$. Surg Endosc 2018; 32: 289-299

[8] von Helden A, Hildenbrand R, Sido B et al. Endoscopic full-thickness resection using an over-the-scope device for treatment of recurrent / residual colorectal neoplasia: a single-center case series. BMC Gastroenterol 2019; 19: 121

[9] Vitali F, Naegel A, Siebler J et al. Endoscopic full-thickness resection with an over-the-scope clip device (FTRD) in the colorectum: results from a university tertiary referral center. Endosc Int Open 2018; 6: E98-E103

[10] Richter-Schrag HJ, Walker C, Thimme R et al. Full thickness resection device (FTRD). Experience and outcome for benign neoplasms of the rectum and colon. Chirurg 2016; 87: 316-325

[11] Velegraki MTA, Vasiliadis K, Fragaki M et al. Endoscopic full-thickness resection of colorectal lesions with the full-thickness resection device: clinical experience from two referral centers in Greece. Ann Gastroeneterol 2019; 32: 1-7

[12] Albrecht $H$, Raithel M, Braun A et al. Endoscopic full-thickness resection (EFTR) in the lower gastrointestinal tract. Tech Coloproctol 2019; 23: 957-963

[13] Bronzwaer MES, Bastiaansen BAJ, Koens L et al. Endoscopic full-thickness resection of polyps involving the appendiceal orifice: a prospective observational case study. Endosc Int Open 2018; 6: E1112-E1119

[14] van der Spek B, Haasnoot K, Meischl C et al. Endoscopic full-thickness resection in the colorectum: a single-center case series evaluating indication, efficacy and safety. Endosc Int Open 2018; 6: E1227E1234

[15] Castor Electronic Data Capture. Amsterdam: 2019: Available from (Accessed: 1 May 2020): https://www.castoredc.com/

[16] Schmidt A, Damm M, Caca K. Endoscopic full-thickness resection using a novel over-the-scope device. Gastroenterology 2014; 147 740-742 e742

[17] Dutch Colorectal Cancer Guideline [In Dutch]. 2014: Available from (Accessed: 7 May 2020): https://richtlijnendatabase.nl/richtlijn/colorectaal_carcinoom_crc/startpagina_-_crc.html

[18] Cotton PB, Eisen GM, Aabakken L et al. A lexicon for endoscopic adverse events: report of an ASGE workshop. Gastrointest Endosc 2010; 71: 446-454

[19] Fujiya M, Tanaka K, Dokoshi T et al. Efficacy and adverse events of EMR and endoscopic submucosal dissection for the treatment of coIon neoplasms: a meta-analysis of studies comparing EMR and endoscopic submucosal dissection. Gastrointest Endosc 2015; 81: 583595

[20] Saito Y, Uraoka T, Yamaguchi Y et al. A prospective, multicenter study of 1111 colorectal endoscopic submucosal dissections (with video). Gastrointest Endosc 2010; 72: 1217-1225

[21] Kuroki Y, Hoteya S, Mitani T et al. Endoscopic submucosal dissection for residual/locally recurrent lesions after endoscopic therapy for colorectal tumors. J Gastroenterol Hepatol 2010; 25: 1747-1753

[22] Hassan C, Repici A, Sharma P et al. Efficacy and safety of endoscopic resection of large colorectal polyps: a systematic review and meta-analysis. Gut 2016; 65: 806-820

[23] Fuccio L, Hassan C, Ponchon T et al. Clinical outcomes after endoscopic submucosal dissection for colorectal neoplasia: a systematic review and meta-analysis. Gastrointest Endosc 2017; 86: 74-86 e17

[24] Arezzo A, Passera R, Marchese N et al. Systematic review and meta-analysis of endoscopic submucosal dissection vs endoscopic mucosal resection for colorectal lesions. United European Gastroenterol ] 2016; 4: 18-29 
[25] Buchner AM, Guarner-Argente C, Ginsberg GG. Outcomes of EMR of defiant colorectal lesions directed to an endoscopy referral center. Gastrointest Endosc 2012; 76: 255-263

[26] Yoshii S, Nojima M, Nosho K et al. Factors associated with risk for colorectal cancer recurrence after endoscopic resection of T1 tumors. Clin Gastroenterol Hepatol 2014; 12: 292-302 e293

[27] Yasue C, Chino A, Takamatsu M et al. Pathological risk factors and predictive endoscopic factors for lymph node metastasis of T1 colorectal cancer: a single-center study of 846 lesions. J Gastroenterol 2019; 54: 708-717
[28] Butte JM, Tang P, Gonen M et al. Rate of residual disease after complete endoscopic resection of malignant colonic polyp. Dis Colon Rectum 2012; 55: 122-127

[29] Richards CH, Ventham NT, Mansouri D et al. An evidence-based treatment algorithm for colorectal polyp cancers: results from the Scottish Screen-detected Polyp Cancer Study (SSPoCS). Gut 2018; 67: 299-306

[30] Williams JG, Pullan RD, Hill J et al. Management of the malignant colorectal polyp: ACPGBI position statement. Colorectal Dis 2013; 15 : (Suppl. 02): 1-38 